\title{
Number Enhancement for Compact Laser-Cooled Atomic Samples by use of Stimulated Radiation Forces
}

\author{
Elizabeth Donley, Tara Cubel Liebisch, Eric Blanshan, and John Kitching \\ Time and Frequency Division \\ National Institute of Standards and Technology \\ Boulder, Colorado 80305 \\ Email: edonley@boulder.nist.gov
}

\begin{abstract}
For cold samples of laser-cooled atoms to be useful in emerging technologies such as compact atomic clocks and sensors, it is necessary to achieve small sample sizes while retaining a large number of cold atoms. Achieving large atom numbers in a small system is a major challenge for producing miniaturized laser-cooled atomic clocks, since the number of captured atoms in a vapor-cell magneto-optical trap (MOT) scales as the fourth power of the laser beam diameter [1]. This strong dependence on size is fundamentally set by the maximum spontaneous light force $\hbar k \gamma / 2$, where $\hbar k$ is the photon momentum and $\gamma / 2$ is the maximum spontaneous photon scatter rate of a saturated transition of linewidth $\gamma$.

We are attempting to surmount the limit imposed by spontaneous emission by using bichromatic cooling [2] - a technique that uses stimulated emission to slow the atoms. We have built a table-top experiment that uses stimulated-emission bichromatic cooling to pre-cool rubidium atoms and dramatically enhance the trappable atom number in a small MOT. The apparatus lets us test how bichromatic cooling scales with miniaturization. Here we report on our first experimental results of cooling a thermal beam of rubidium atoms down to MOT capture velocities.
\end{abstract}

\section{INTRODUCTION}

DARPA's new Integrated Micro Primary Atomic Clock Technology Program (IMPACT) has set a goal to deliver the performance of a cesium beam clock in a device that is $1 / 1000^{t h}$ as large in terms of size and consumed power. The performance specs for the IMPACT program require the frequency instability to continue to average down for time scales out to a month and achieve a time uncertainty of $32 \mathrm{~ns}$ in one month $\left(1.2 \times 10^{-14}\right)$. In contrast to that, the ChipScale Atomic Clock Program (CSAC) specified a frequency instability of $1 \times 10^{-11}$ at one hour but did not specify a stability metric at longer times. To achieve the ambitious goals of the IMPACT program, the main sources of drift for CSAC clocks have to be eliminated. One cause of drift is the buffer gas shift, which can be eliminated by interrogating laser-cooled atoms in vacuum. (For a review of CSACs and sources of their drift, see [3]).

While interrogation of laser-cooled samples has the advantage of eliminating buffer gas shifts, it has the disadvantage that the number of MOT atoms decreases rapidly with smaller beam size. The MOT atom number versus beam size is

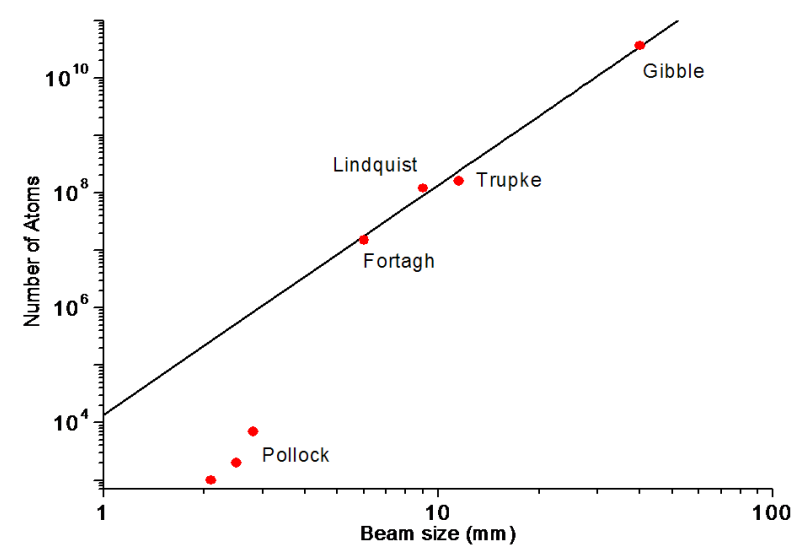

Fig. 1. MOT atom number versus beam size. Experimental measurements are shown as red symbols and the theoretical curve is calculated from the expression in the text. From large to small, the references for the measurements are Gibble: [5], Trupke: [6], Lindquist: [4], Fortagh: [7] and Pollock: [8]. The Trupke and Pollock measurements were performed using silicon pyramidal traps, and for those measurements the beam diameter is set equal to the pyramid depth. The Gibble and Lindquist measurements were performed using traditional vapor-cell configurations. The MOT for the Fortagh measurement was directly loaded with a $\mathrm{Rb}$ dispenser.

shown in Fig. 1 for a number of experiments, along with the theoretical dependence that is known to agree well with experiments for large laser beams [1], [4]:

$$
N=\frac{0.1 \pi d^{4} r^{2} v_{r c}}{4 \sigma v_{a v g}^{4}} .
$$

Here $d$ is the beam diameter, $r$ is the photon scatter rate, $v_{r c}$ is the recoil velocity, $\sigma$ is the cross section for a background collision that ejects an atom from the trap, and $v_{a v g}$ is the average velocity. While the model works well for MOT sizes over $6 \mathrm{~mm}$, the number falls off for the measurements done with smaller beam sizes. Pollock and colleagues suggest that for small MOTs the atom number falls off even more sharply than the $d^{4}$ scaling, and may scale as $d^{6}$ [8].

For a laser-cooled atomic clock, the number of atoms capturable in the MOT sets a limit on the frequency stability 
via the quantum projection noise [9]:

$$
\sigma_{y}(\tau)=\frac{1}{2 \pi T_{R} \nu_{0}} \sqrt{\frac{T_{c}}{\tau}} \frac{1}{\sqrt{N}} .
$$

Here $T_{R}$ is the Ramsey period, $\nu_{0}$ is the resonance frequency, $T_{c}$ is the cycle period, $\tau$ is the integration period, and $N$ is the number of interrogated atoms. The expression in Eq. 2 includes only the quantum projection noise and neglects terms from photon shot noise, technical noise, and noise from the local oscillator. The IMPACT program goal is to achieve an instability of $1 \times 10^{-11}$ at one second of integration. Given the additional noise terms, we anticipate that the instability related to the quantum projection noise should be $1 \times 10^{-12}$ at one second. Assuming values of $T_{c}=100 \mathrm{~ms}$ and $T_{R}=10 \mathrm{~ms}$, this requires $10^{6}$ atoms to reach the desired stability. Given the relatively short cycle period of $100 \mathrm{~ms}$, this would imply a steady-state atom number near $1 \times 10^{7}$, which would require 5 $\mathrm{mm}$ laser beams. This is not small by our standards. Increasing the number of steady-state atoms that can be trapped will always reduce technical noise, since more atoms allow us to optimize the trade off between fast load periods and low background scattered light.

The number of atoms trappable in a vapor cell MOT is fundamentally limited by the photon scattering rate $r$, whose maximum value is limited to $\gamma / 2$ for the case of laser cooling by spontaneous emission, where $\gamma=2 \pi \times 6.065 \mathrm{MHz}$ is the natural linewidth for $\mathrm{Rb}$. We are attempting to beat the limit imposed by spontaneous emission by cooling the atoms with stimulated emission, for which there is no upper limit to the photon scatter rate. More specifically, we intend to use a technique called bichromatic cooling [2]. In an early demonstration of the technique, Söding et al were able to decelerate the longitudinal velocity of a large fraction of the atoms in an atomic beam from thermal velocities down to velocities that are capturable in a MOT. This bichromatic cooling experiment achieved cooling forces that were $10 \times$ larger than the limit imposed by spontaneous emission. Bichromatic cooling has also been studied extensively in Hal Metcalf's group and has been used to deflect atomic beams of rubidium [10] and collimate a beam of metastable helium atoms [11]. We intend to use the technique to slow atoms in an atomic beam and capture them in a millimeter-scale MOT, ultimately exceeding the number of atoms that would have been captured had the MOT been loaded from the background vapor. We also intend to test how the method scales with miniaturization using lower power, shorter cooling distances, and smaller-diameter laser beams.

\section{BICHROMATIC COOLING}

1) Background: Fig. 2 conceptually illustrates the bichromatic cooling effect [2]. An atom with velocity $v_{\text {atom }}$ is traveling from left to right. A bichromatic laser beam strikes the atom from the right with frequency components $\omega_{0}-k v_{a t o m} \pm$ $\Delta$ and second bichromatic laser beam strikes the atom from the left with frequency components $\omega_{0}+k v_{a t o m} \pm \Delta$. The two frequency components in the beams from each side interfere

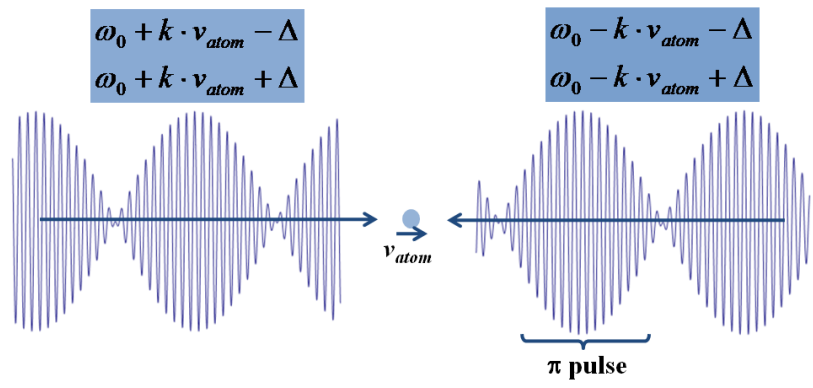

Fig. 2. The bichromatic cooling scheme.

to produce pulse trains. We can set the parameters of the frequency components such that each beat produces a $\pi$ pulse by setting the relationship between the Rabi frequency $\Omega_{R}$ and the detuning $\Delta$ such that $\Omega_{R}=(\pi / 4) \Delta$. Here $\Omega_{R}=$ $\gamma \sqrt{I /\left(2 I_{\text {sat }}\right)}$ is the Rabi frequency for a single frequency component, where $I_{\text {sat }}$ is the saturation intensity. For the pulse trains to cool the atoms, the phase has to be set so that the atoms absorb photons from the leftward traveling beam and emit them into the rightward traveling beam, thus slowing them by two photon momenta per stimulated-emission cycle. For optimal forces, the phase difference between the pulse trains is set such that $|\phi| \simeq \pi / 2$. This phase shift is a compromise between producing forces in the preferred direction and minimizing the overlap of the pulses. For the case of the rightward-traveling atom in Fig. 2, the leftward traveling pulse train leads (lags) the rightward traveling pulse train by $\pi / 2$ for cooling (heating).

2) Our Bichromatic Cooling Apparatus: Fig. 3A is a photo of our vacuum chamber. At the top of the chamber is the $\mathrm{Rb}$ source, which is separated from the detection chamber by an atomic beam collimator shown in the inset. The atomic beam flux and velocity distribution are set by the temperature of the $\mathrm{Rb}$ source chamber, which is heated to as high as $140^{\circ} \mathrm{C}$ with the addition of heat tapes to the chamber. The collimator is mounted in a $70 \mathrm{~mm}$ conflat flange in the center of the apparatus, and consists of two apertures of $0.5 \mathrm{~mm}$ diameter separated by $2.5 \mathrm{~cm}$. The laser beams enter the chamber from above and below through windows along the axis of the atomic beam. The beams are focused down by lenses of $1 \mathrm{~m}$ focal length outside of the chamber and pass through the collimator apertures along with the atomic beam. To minimize absorption from high density thermal background atoms in the oven, there is a long inverted window in the $\mathrm{Rb}$ oven that limits the path length of the laser beams to a few millimeters in the high-density region. Each frequency component typically has a power of two milliwatts, which gives a typical Rabi frequency of $17 \gamma$. Note that our cooling power per beam is about a factor of $1 / 20^{\text {th }}$ of that used in [2] and our apertures are $1 / 4^{\text {th }}$ as large. Our values of $\Delta$ are typically about $2 \pi \times 100 \mathrm{MHz}$.

Repump and probe laser beams are superimposed on one of the cooling beams with the same circular polarization as the cooling light. The repump beam has about $3 \mathrm{~mW}$ of power 

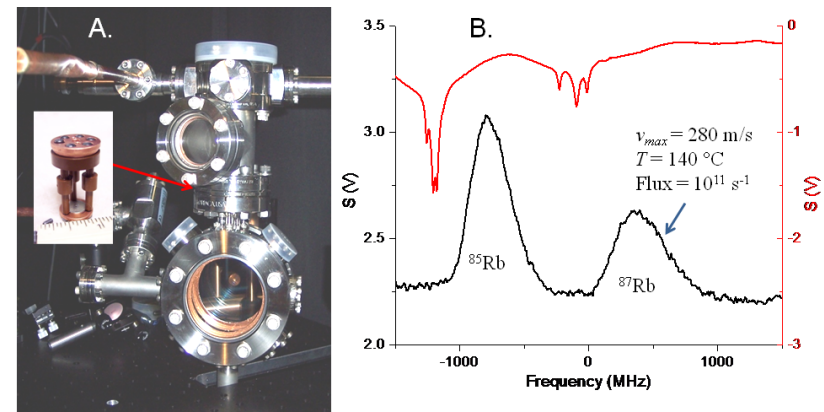

Fig. 3. A. A picture of our bichromatic cooling apparatus. The atomic beam collimator is shown in the inset. B. Velocity distributions measured with our apparatus (black) and a saturated absorption signal used for calibration (red).

and its spectrum is broadened to cover the whole absorption profile of the ${ }^{87} \mathrm{Rb}$ velocity distribution by modulating the laser current with white noise. The probe beam power is approximately $100 \mu \mathrm{W}$ in power. The probe-beam fluorescence is detected by a photomultipler tube through one of the large windows in the detection chamber. The probe and cooling beams are chopped on and off with acousto-optic modulators on the time scale of several microseconds such that the cooling light is off when probing and the probe light is off while cooling. The fluorescence measured by the photomultiplier tube is integrated only while probing. The duty cycle of the cooling/probing sequence can be changed to vary the effective cooling length, enabling tests of how the method scales with miniaturization. The effective cooling length with a duty cycle of $50 \%$ is about $3 \mathrm{~cm}$ (30\% of the cooling distance used in [2]). The frequencies of the cooling light are produced with a combination of three acousto-optic modulators.

Fig. 3B shows plots of the measured velocity distributions (left axis) and a reference saturated absorption signal for frequency calibration (right axis). Because our $\mathrm{Rb}$ source contains a sample of natural abundance, we see both common isotopes of $\mathrm{Rb}$ in the spectrum. The horizontal axis indicates the probe frequency. The zero of the frequency axis is set equal to the frequency of the ${ }^{87} \mathrm{Rb}$ cycling transition for atoms at rest. The saturated absorption spectrum is freqeuncy shifted from the atom beam spectrum by an offset introduced by an acousto-optic modulator that is used to switch the probe beam light on and off.

\section{Bichromatic CoOling MeAsurements}

Although many parameters remain to be optimized and improved in our apparatus, we have begun to see bichromatic force signals with our system. Fig. 4A shows measured velocity distributions with the bichromatic beams on and off with the bichromatic beat phase set such that the atoms were sped up. A calculation of the expected force profile for our experimental parameters is also shown (right axis) [12].

When the bichromatic light is on, a peak appears in the velocity distribution where we would expect - atoms are being pushed to higher velocity from the region of the velocity distribution that is acted on by the force profile. From the

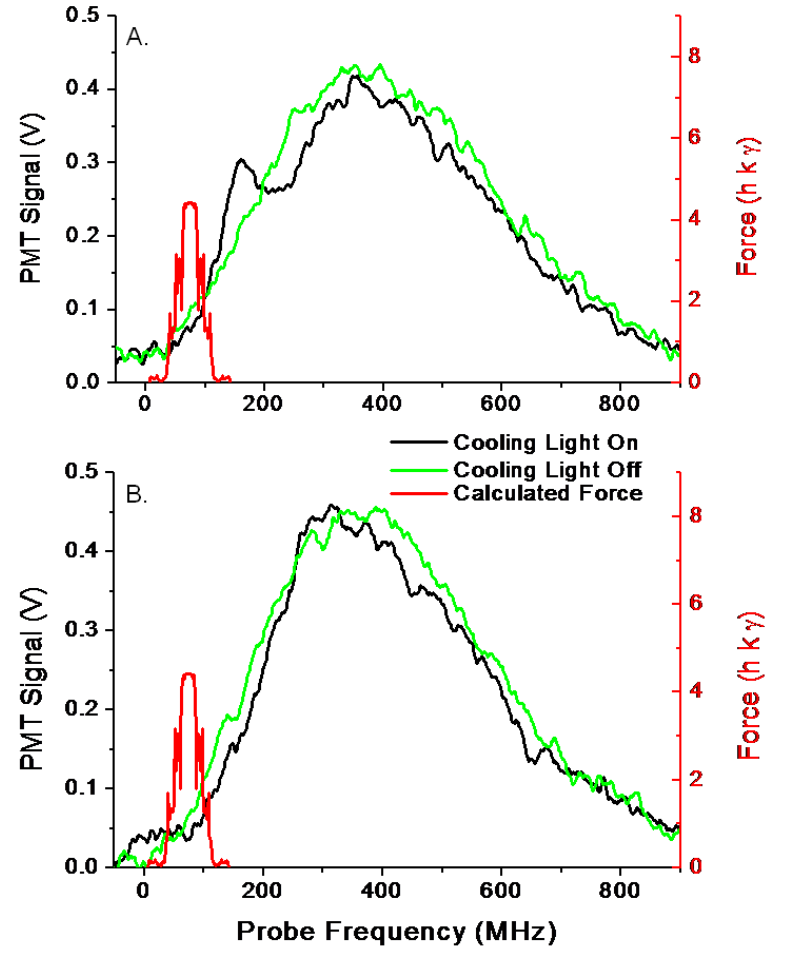

Fig. 4. Measured velocity distributions with and without the bichromatic cooling beams on (left axis). The expected force profile for our experimental parameters is also shown (right axis). A.) Measured distributions with the beat phase for the bichromatic cooling beams set to speed up the atoms. B.) Measured distributions with the beat phase set to produce slowing. Note that some of the atoms have negative velocity in the detection region.

area under the peak of faster atoms, we estimate that the bichromatic light is addressing and speeding up about $2 \%$ of the total detected atoms.

While the peak of higher-velocity atoms is quite prominent, there is not a dip of equal area being carved in the distribution under the force profile. We believe that this is a detection artifact in our apparatus. While the cooling and probe beams are well mode-matched to the atomic beam within the collimator, the atomic beam diverges more rapidly outside of the collimator such that the probe beam overlaps only with the central portion of the atomic beam beyond the apertures. In the detection region, the atomic beam radius is about $1.2 \mathrm{~mm}$, while the probe beam radius is only about $0.2 \mathrm{~mm}\left(1 / e^{2}\right)$. Only atoms that have sufficiently low values of $v_{r} / v_{z}$ are detected, where $v_{r}$ is the radial atom velocity and $v_{z}$ is the longitudinal atom velocity. Heating and/or cooling atoms alters the detected sample by changing $v_{z}$. By increasing $v_{z}$, some atoms are detected that would have been missed. By reducing $v_{z}$, we increase the divergence angle for slowed atoms and fail to detect them with our current probe configuration. Note that this is not a fundamental problem, but is only a result of experimental constraints in our system that we will work to correct.

Fig. 4B shows the effect on the velocity distribution when 
phase is adjusted to produce slowing. Even though we are missing some of the slowed atoms in our detection system, we estimate a factor of 10 increase in atoms with velocities between $0<v<13 \mathrm{~m} / \mathrm{s}$ with the cooling light on. For a MOT with $1 \mathrm{~mm}$ diameter laser beams, $13 \mathrm{~m} / \mathrm{s}$ is the capture velocity based on spontaneous-force deceleration.

\section{OUTLOOK}

Our apparatus is beginning to produce stimulated-force changes in the atomic velocity distributions, but many parameters need to be optimized. High on our agenda is to optimize the cooling and detection optics and load the atoms into an optical molasses, the efficiency of which will provide a metric to characterize the slowing. We will also characterize the number enhancement versus cooling distance.

\section{ACKNOWLEDGMENT}

We are grateful for many fruitful discussions with our colleagues Jenni Strabley and Ken Salit. We acknowledge funding from NIST and DARPA, and T.C.L. acknowledges support from the NRC. This paper is a contribution of the National Institute of Standards and Technology, which is an agency of the U.S. government, and is not subject to copyright.

\section{REFERENCES}

[1] C. Monroe, W. Swann, H. Robinson, and C. Wieman, "Very cold trapped atoms in a vapor cell," Phys. Rev. Lett. vol. 65, 1571-1574 (1990)

[2] J. Söding, R. Grimm, Y.B. Ovchinnikov, P. Bouyer, and C. Salomon, "Short-distance atomic beam deceleration with a stimulated light force," Phys. Rev. Lett. vol. 78, 1420-1423 (1997)
[3] S. Knappe, "MEMS Atomic Clocks," Comp. Microsys. vol. 3, 571-612 (2007)

[4] K. Lindquist, M. Stephens, and C. Wieman, "Experimental and theoretical study of the vapor-cell Zeeman optical trap," Phys. Rev. A vol. 46, 40824090 (1992)

[5] K.E. Gibble, S. Kasapi, and S. Chu, "Improved magneto-optic trapping in a vapor cell," Opt. Lett. vol. 17, 526-528 (1992)

[6] M.Trupke, R. Ramirez-Martinez, E.A. Curtis, J.P. Ashmore, S. Eriksson, E.A. Hinds, Z. Moktadir, C. Gollasch, M. Kraft, G. Vijaya Prakash, and J.J. Baumberg, "Pyramidal micromirrors for microsystems and atom chips," Appl. Phys. Lett. vol. 88, 071116, 2006

[7] J. Fortagh, A. Grossmann, T.W. Hänsch, and C. Zimmermann, "Fast loading of a magneto-optical trap from a pulsed thermal source," $J$. of App. Phys. vol. 84, 6499-6501 (1998)

[8] S. Pollock, J.P. Cotter, A. Laliotis, and E.A. Hinds, "Integrated magnetooptical traps on a chip using silicon pyramid structures," Opt. Express vol. 17, 14109-14114 (2009)

[9] G. Santarelli, Ph. Laurent, P. Lemonde, A. Clairon, A.G. Mann, S. Chang, A.N. Luiten, and C. Salomon, "Quantum projection noise in an atomic fountain: A high stability cesium frequency standard," Phys. Rev. Lett. vol. 82, 4619 (1999).

[10] M.R. Williams, F. Chi, M.T. Cashen, and H. Metcalf, "Measurement of the bichromatic optical force on Rb atoms," Phys. Rev. A vol. 60, R1763-R1766 (1999)

[11] M. Partlow, X. Miao, J. Bochmann, M. Cashen, and H. Metcalf, "Bichromatic slowing and collimation to make an intense helium beam," Phys. Rev. Lett. vol. 93, 213004 (2004)

[12] The computer code used for the calculation solves the optical Bloch equations and was kindly provided to us by Johannes Söding. 